Arq. Bras. Med. Vet. Zootec., v.68, n.1, p.48-56, 2016

\title{
Percepção de equipes laboratoriais quanto a questões de bem-estar animal
}

\author{
[Perception of laboratory staff regarding animal welfare issues] \\ B.G.F. Deguchi, P.R. Tamioso, C.F.M. Molento* \\ Universidade Federal do Paraná - UFPR- Curitiba, PR
}

\begin{abstract}
RESUMO
As equipes laboratoriais controlam diversas características do ambiente dos animais utilizados em pesquisas. Portanto, suas atitudes têm grande influência no bem-estar animal (BEA) e nos resultados obtidos. Buscou-se verificar o conhecimento e a percepção dessas equipes em relação a questões de BEA. Os dados foram coletados por meio de um questionário online composto por 22 questões abertas e 23 fechadas. As respostas são referentes a 62 participantes de diversas instituições de pesquisa brasileiras. Dezesseis $(25,8 \%)$ não receberam treinamento para exercer suas funções, e $11(18,0 \%)$ realizavam ou coordenavam procedimentos com potencial para causar dor ou morte. O principal fator limitante relatado para o uso de animais em pesquisas foi referente a questões éticas $(38 ; 63,3 \%)$. Todos declararam conhecer o significado do termo BEA; porém, a maioria dos conceitos expressos foi de forma parcial (32; $64,0 \%$ ). Tais resultados podem estar relacionados ao caráter optativo ou à indisponibilidade do ensino de BEA na maioria dos cursos de graduação no Brasil. Os animais vertebrados foram percebidos pelos respondentes como portadores de alto grau de senciência. Espécies em contato social e afetivo com os seres humanos foram vistas como mais sencientes que outros grupos. O número de respondentes interessados em um projeto de enriquecimento ambiental $(34 ; 69,4 \%)$ sugere preocupação com o BEA. Os resultados apresentados podem subsidiar a localização de pontos críticos de BEA em laboratórios brasileiros e indicam possibilidades para melhoria no conhecimento científico de questões centrais relativas ao BEA.
\end{abstract}

Palavras-chave: animais usados em laboratório, biotério, experimentação animal, Lei Arouca

\begin{abstract}
Laboratory staff controls a series of environmental parameters affecting animals used in research. Therefore, staff attitudes influence both animal welfare $(A W)$ and research results. This study aimed to verify the knowledge and perception of staff members on AW. Data were collected through a 22 openquestion and 23 multiple-choice question online survey. 62 respondents from Brazilian institutions answered the survey. Sixteen (25.8\%) participants did not receive training for their functions, from which $11(18.0 \%)$ performed or coordinated procedures that may induce pain or death. The main limiting factor for the use of animals in research was ethical issues $(38,63.3 \%)$. All participants reported to know the meaning of $A W$, but most of the concepts given were partial (32, 64.0\%). These results may be related to the unavailable or optional teaching of AW in most undergraduate courses in Brazil. Vertebrates were perceived by respondents as highly sentient. Species with a social and affective bond with human beings were seen as more sentient than other groups. There was interest in an environmental enrichment project (34, 69.4\%) suggesting concern with AW. These results can collaborate in the identification of critical issues in AW in Brazilian laboratories and indicate opportunities to improve scientific knowledge of key issues related to $A W$.
\end{abstract}

Keywords: laboratory animals, animal facilities, animal research, Arouca Law.

Recebido em 22 de abril de 2015

Aceito em 5 de outubro de 2015

*Autor para correspondência (corresponding author)

E-mail: carlamolento@ufpr.br 


\section{INTRODUÇÃO}

A utilização de animais para pesquisas é recorrente em várias áreas do conhecimento científico. A validade e a confiabilidade de tais estudos são maximizadas quando materiais, animais e métodos são seguros e reproduzíveis. Os animais usados em laboratório são influenciados por diversos parâmetros ambientais: temperatura, umidade, sons, agentes químicos, luz e outros animais, incluindo o ser humano (Baumans, 2010). Assim, conhecer as características das variáveis que influenciam a qualidade de vida dos animais é determinante para se obter resultados confiáveis e defensáveis do ponto de vista ético.

Os efeitos do manejo e do ambiente nas funções fisiológicas e psicológicas por vezes não são percebidos; no entanto, afetam os resultados (Faith e Huerkamp, 2009). Há evidências indicando que as condições de manutenção de animais usados em laboratório podem mascarar diferenças genéticas e induzir comportamentos anormais; com isso, experimentos iguais podem apresentar resultados diferentes (Bohannon, 2002). A interpretação de tais efeitos é essencial, uma vez que somente é possível exercer controle de variáveis cujos efeitos sejam compreendidos.

Atualmente, estima-se a criação de cerca de 115,3 milhões de animais vertebrados ao ano para a experimentação em todo o mundo (Taylor et al, 2008). Os animais são mantidos sob condições padronizadas, consideradas ideais para a utilização em pesquisas, mas que com frequência não permitem o suprimento das necessidades dos animais, em especial aquelas comportamentais e psicológicas. Assim, em geral, há comprometimento do grau de bem-estar dos animais. Tal situação tem implicações científicas, na medida em que diminui a qualidade dos resultados, e éticas, na medida em que diminui o grau de bem-estar dos animais.

A padronização dos métodos em experimentação é, em geral, altamente controlada. No entanto, características relevantes das equipes laboratoriais que afetam o bem-estar animal necessitam ser conhecidas. O objetivo deste trabalho foi diagnosticar o conhecimento dos indivíduos que trabalham nos laboratórios e biotérios sobre questões básicas de bem-estar de animais de laboratório.

\section{MATERIAL E MÉTODOS}

O estudo foi aprovado pelo Comitê de Ética em Pesquisa do Setor de Ciências da Saúde da Universidade Federal do Paraná sob $n^{\circ}$ CAAE 0175.0.0.091.000-11. Os respondentes foram integrantes de equipes laboratoriais e de biotérios, sendo técnicos, pesquisadores e coordenadores. Foram enviados contatos individuais e também aos integrantes da lista eletrônica Redusal/Biotbras, do Centro Multidisciplinar para Investigação Biológica na Área da Ciência em Animais de Laboratório (Cemib), Unicamp. Atualmente a referida lista eletrônica é composta por cerca de 900 inscritos do Brasil e de diversos outros países. O questionário foi criado na plataforma Google.docs ${ }^{\circledR}$. Os convidados receberam uma mensagem eletrônica que os encaminhou para o preenchimento do formulário online.

O questionário completo continha 22 questões abertas e 23 fechadas, totalizando 45 questões, sendo as principais: Você recebeu treinamento para exercer suas atividades?; Quais as funções das Comissões de Ética no Uso de Animais (CEUAs)?; Os projetos que você participa foram submetidos à CEUA?; Realiza ou coordena algum procedimento que cause sofrimento ou morte de animais?; Qual a sua percepção de senciência entre espécies?; Há algum limite que não devemos ultrapassar quando animais são usados na experimentação? Adicionalmente, houve perguntas sobre conceitos e legislação de bem-estar animal. As questões fechadas foram dicotômicas (sim/não) ou em escala Likert. Para as respostas abertas, foi utilizada a análise de conteúdo com a separação dos diferentes grupos de respostas. As respostas relativas aos conceitos apresentados sobre senciência animal, 3Rs da experimentação, BEA, cinco liberdades, EA e Lei $11.794 / 2008$ foram categorizadas como satisfatórias, parciais ou insatisfatórias.

Todas as variáveis foram submetidas à análise estatística descritiva. Nas questões sobre o grau de senciência em diferentes espécies e limites para experimentação, foram realizados os testes de Mann-Whitney e Kruskal-Wallis com a correção de Bonferroni. 


\section{Deguchi et al.}

\section{RESULTADOS E DISCUSSÃO}

Foram recebidos 62 questionários respondidos, provenientes de 33 instituições das regiões Norte (quatro; 6,5\%), Nordeste (sete; 11,3\%), Sudeste $(15 ; 24,2 \%)$ e Sul $(33 ; 53,2 \%)$ do Brasil, sendo $17(27,4 \%)$ respondentes do sexo masculino e 45 $(72,6 \%)$ do sexo feminino. Os participantes declararam escolaridade de nível fundamental (um; $1,6 \%$ ), nível médio (sete; $11,3 \%$ ), superior em Medicina Veterinária (24; 38,7\%), Ciências Biológicas (21; 33,9\%), Farmácia e Bioquímica (quatro; 6,5\%), Psicologia (dois; 3,2\%) e outros cursos de nível superior (três; 4,8\%). Os cursos superiores foram realizados em instituições públicas $(38 ; 61,3 \%)$ e particulares $(16 ; 25,8 \%)$, nos períodos: até o ano $2000(20 ; 32,3 \%)$, do ano 2000 ao de $2008(20 ; 32,3 \%)$ e de 2008 a 2012 $(13 ; 21,0 \%)$. A descrição que apresentaram de seus cargos foi de coordenadores, supervisores ou diretores $(13 ; 20,0 \%)$, médicos veterinários (quatro; 6,2\%), pesquisadores $(19 ; 29,2 \%)$, responsáveis técnicos $(10 ; 15,4 \%)$ e técnicos $(19$; $29,2 \%)$. Dezesseis $(25,8 \%)$ dos participantes não receberam treinamento para exercer suas funções nos laboratórios. Aqueles que realizavam ou coordenavam procedimentos que podem causar dor e morte representam 51,6\% (32) dos participantes. Os procedimentos realizados foram a eutanásia $(35,5 \% ; 22)$, a indução de doenças e ferimentos $(16,1 \%, 10)$, a realização de cirurgias $(8,1 \%$; cinco), a coleta de materiais in vivo $(8,1 \%$; cinco), a administração de fármacos $(4,8 \%$; três) e a indução de estresse (1,6\%; um). Alguns respondentes que realizavam ou coordenavam tais procedimentos não receberam nenhum treinamento $(11 ; 18,0 \%)$. Aqueles que receberam treinamento $(46 ; 74,2 \%)$ realizaramno com outros integrantes dos laboratórios (22; $35,5 \%$ ), em instituições de ensino superior no Brasil (16; 25,8\%), em disciplinas da graduação, pós-graduação e sem especificação (cinco; $8,1 \%$ ), na Fundação Oswaldo Cruz (quatro; $6,5 \%$ ), em instituições fora do Brasil (quatro; $6,5 \%$ ), na Sociedade Brasileira de Ciências em Animais de Laboratório (três; $4,8 \%$ ) ou pelo Conselho Federal de Medicina Veterinária (dois; $3,2 \%$ ). A interpretação individual sobre o significado do termo treinamento foi ampla. É possível que alguns considerem palestras e disciplinas uma forma de treinamento, enquanto outros considerem apenas cursos especializados e acompanhamento no local de trabalho como formas de treinamento. Dos participantes que afirmaram ter realizado treinamento, $17,7 \%$ (11) indicaram algum curso específico com animais usados em laboratório. $\mathrm{O}$ aprendizado informal foi a principal forma mencionada. $\mathrm{O}$ treinamento dos profissionais que exercem ou exercerão práticas com os animais pode definir a eficiência de suas atribuições. Para lidar com as constantes mudanças no conhecimento e apresentá-las de forma correta, é necessário o treinamento nas ciências de animais usados em laboratório e em BEA, assim como educação continuada (Nevalainen, 2007). Havendo um programa de treinamento formalizado constituído de cursos específicos, pode-se obter um melhor padrão dos conteúdos que são apresentados, com atualização eficiente. Segundo o Canadian Council on Animal Care (1999), o propósito precípuo de qualquer programa de treinamento deve ser promover a compaixão e o respeito aos animais e, em segundo lugar, transmitir a base teórica e as habilidades técnicas para realização dos procedimentos.

A maioria dos participantes indicou conhecer a Lei Arouca (46; 74,2\%). Na avaliação do conhecimento da referida lei, $45,2 \%$ (28) das declarações foram consideradas satisfatórias, $19,4 \%$ (12) parciais, e nenhuma insatisfatória. Verificou-se que os profissionais em contato direto com animais usados em laboratório possivelmente têm maior contato com a legislação que alunos, porém, quanto à real compreensão da lei, não há amplo conhecimento. Alunos brasileiros de graduação em Ciências Biológicas, Farmácia, Medicina e Medicina Veterinária apresentaram pouco conhecimento da referida lei; $54,5 \%$ a conheciam, não havendo diferenças entre calouros e veteranos (Deguchi et al., 2012). Com a aprovação da referida lei, tornou-se obrigatória a presença das CEUAs em todas as instituições que utilizam animais, assim como a submissão dos projetos com animais à CEUA (BRASIL, 2008). Entre os respondentes, $90,7 \%$ (49) indicaram que, nos projetos de que participaram, haviam sido submetidos a uma CEUA, e 9,3\% (cinco) que tal medida não fora realizada. Alguns participantes relataram não terem acesso a um formulário de submissão de projeto à CEUA (nove; 15,8\%).

As funções das Comissões de Ética no Uso de Animais (CEUAs) indicadas pelos participantes foram, em sua maioria, condizentes com a interpretação tradicional da legislação (49; 
$62,8 \%$ ). Entretanto, outras funções também foram indicadas $(12 ; 15,4 \%)$, como, por exemplo, a realização de atividades educativas e de extensão à sociedade. A opinião sobre a necessidade de ações fiscalizatórias (12; 15,4\%) e a promoção de melhores condições de BEA (cinco; 6,4\%) também foram indicadas. Adicionalmente, nos comentários foram expostas novamente questões relacionadas à fiscalização (11, 17,7\%), críticas positivas (nove; 14,5\%), críticas negativas (sete; 11,3\%) e outros comentários (nove; 14,5\%). Apenas um participante $(1,6 \%)$ indicou que a verificação do mérito científico da pesquisa deveria ser função da CEUA. O julgamento do mérito científico pela CEUA possibilita uma melhor análise dos projetos, uma vez que verifica se o estudo em questão se justifica.

A fiscalização é um dos pontos mais discutidos com relação à ação das CEUAs. Na legislação federal não há indicação explícita de que as CEUAs devam agir na físcalização das pesquisas. Entretanto, a legislação estadual exige a ação de fiscalização dessas atividades pelas CEUAs nos estados do Rio de Janeiro (Lei no. 3900), Paraná (Lei no. 14037), Santa Catarina (Lei no. 12854), Rio Grande do Sul (Lei no. 1191) e São Paulo (Lei no. 11977). A fiscalização não é um procedimento fácil, devido à dinâmica das comissões que atuam geralmente como uma atividade adicional não prioritária (Schuppli e Fraser, 2007). É necessária uma discussão mais ampla sobre como monitorar a experimentação e como tal trabalho pode ser efetivado.

As considerações éticas relacionadas aos animais foram citadas como um dos principais limitantes ao uso de animais para experimentação (38; 63,3\%). Aproximadamente $320 \quad(33,0 \%)$ pesquisadores de diversos países indicaram que mantêm alguma preocupação ética quando animais são utilizados em pesquisas (Cressey, 2011), sugerindo maior preocupação nos respondentes brasileiros. Por outro lado, no Paraná, professores e estudantes de Medicina Veterinária não declararam o sofrimento animal como uma desvantagem de seu uso no ensino (Zanetti et al., 2011). As questões éticas com foco no animal não foram citadas pelo restante dos participantes, que dividiram suas opiniões entre questões de ética geral $(10 ; 16,7 \%)$, metodologia (oito; 13,3\%) e inexistência de quaisquer limites para o uso de animais (quatro; $6,7 \%$ ), evidenciando a necessidade de melhoria da reflexão sobre ética animal.

Apesar de apenas quatro (6,7\%) respondentes indicarem que não existem limites para o uso de animais em experimentos (quatro; 6,7\%), tais respostas têm alta relevância, devido ao efeito que podem gerar sobre o BEA. A falta de reconhecimento de um limite no uso de animais sugere um pensamento cartesiano obsoleto no que tange à compaixão com animais, uma vez que há amplo reconhecimento da ciência atual de que várias espécies de animais apresentam estados de consciência (Low et al., 2012). Em relação às espécies de animais utilizados, as mais citadas pelos respondentes foram ratos (35; $37,2 \%)$, camundongos $(33 ; 35,1 \%)$, coelhos (sete; 7,4\%), peixes (cinco; 5,3\%), hamsters (quatro; 4,3\%), cobaias (quatro; 4,3\%), macacos (três; 3,2\%), aves (um; 1,1\%), invertebrados (um; $1,1 \%$ ) e gambás (um; $1,1 \%$ ). Os resultados indicam os ratos e os camundongos como os animais mais utilizados na experimentação, seguindo a tendência mundial (Baumans, 2005). A preferência do uso de camundongos e ratos em experimentação pode ser relativa à genética desses animais, que é amplamente conhecida e manipulada, a seu tamanho corporal reduzido, aos ciclos de vida e de reprodução curtos e à proximidade taxonômica em relação aos seres humanos (Baumans, 2010).

Alguns países mantêm dados oficiais sobre o número de animais utilizados na experimentação, embora haja possibilidade de que estejam subestimados devido à legislação de cada país. No Brasil, tais informações não estão disponíveis. Pesquisas indicam que o Brasil utilizou cerca de 1,16 milhão de animais em 2005, localizando-se na nona posição de países quanto ao número de animais utilizados (Taylor et al., 2008). Em 2006, em levantamento baseado em artigos científicos que continham o número de animais utilizados em 18 revistas científicas do estado do Paraná, foram contabilizados 3.497.653 animais, sendo 216.223 vertebrados (Silla et al., 2010). Permanece a necessidade de se organizarem e se disponibilizarem informações sobre espécies mais utilizadas, número de indivíduos e tipos de procedimentos realizados no Brasil. 
De acordo com os participantes, o destino dos animais utilizados nas pesquisas é a eutanásia (54; $85,7 \%)$, a eutanásia e subsequente utilização no ensino (três; 4,8\%), o encaminhamento a outro experimento (três; 4,8\%), a adoção (dois; $3,2 \%$ ) e o tratamento do animal após o experimento (um; 1,6\%). Nos casos de adoção, os animais podem ser destinados a entidades protetoras de animais ou a pessoas idôneas quando os critérios de biossegurança forem respeitados (BRASIL, 2008). Esta é uma prática que vem ganhando adeptos; indivíduos das espécies com as quais os seres humanos têm maior compaixão, principalmente o cão, são aqueles mais adotados (Beagle Freedom Project, 2012). Segundo Webster (2005), o cuidado com os animais está comumente relacionado ao seu valor extrínseco; por exemplo, há diferença no tratamento dado a um rato de estimação e a outro usado em laboratório.

As vantagens percebidas do uso de animais em pesquisa foram a contribuição para ciência e tecnologia $(18 ; 25,7 \%)$, a representação de um organismo complexo $(15 ; 21,4 \%)$, a extrapolação dos estudos para os seres humanos $(14 ; 20,0 \%)$, o controle experimental $(10 ; 14,3 \%)$, o menor tempo de estudo necessário para se obter resultados (cinco; 7,1\%), o maior número amostral possível (três; 4,3\%), a fácil manutenção (três, 4,3\%) e o baixo custo (dois; $2,9 \%$ ). As desvantagens foram referentes ao sofrimento animal $(20 ; 37,0 \%)$ e outras considerações não relacionadas ao sofrimento (32; 59,3\%); segundo dois respondentes $(3,7 \%)$, não havia qualquer desvantagem. A falta de percepção de que o sofrimento animal é uma desvantagem pode indicar processos anteriores de dessensibilizacão, no sentido de diminuição da preocupação com os animais. A adoção de procedimentos psicologicamente desagradáveis tende à dessensibilização daqueles que realizam ou presenciam tais técnicas (Tréz, 2011).

Além da pesquisa, os participantes também indicaram para uso justificável com animais: o ensino $(12 ; 18,8 \%)$, as situações para as quais não existam métodos alternativos $(11 ; 17,2 \%)$, qualquer uso em prol da saúde humana (nove; $14,1 \%$ ), a alimentação (oito; $12,5 \%$ ), a utilização de animais de tração (sete; 10,9\%), as terapias com animais (cinco; 7,8\%), os testes de controle de qualidade de produtos (quatro; 6,3\%), a guarda e a proteção (três; 4,7\%), as pesquisas comportamentais (três; 4,7\%) e como animal de estimação (dois; $3,1 \%$ ). Nos usos relacionados à interação social com animais, foi recorrente a indicação de que a utilização deve ser de forma a respeitá-los e a promover o seu bem-estar. Segundo Webster (2005), a preocupação com o bem-estar animal demonstra a separação de valores entre os animais com forte vínculo social com o ser humano e aqueles sem a mesma proximidade.

A maioria dos entrevistados indicou que conhece os 3Rs $(46 ; 74,2 \%)$, com a predominância de conceituação satisfatória $(34 ; 77,3 \%)$, seguida de $18,2 \%$ (oito) parciais e $4,5 \%$ (dois) insatisfatórias. Os 3Rs da experimentação são amplamente difundidos na literatura científica e nas legislações relacionadas (BRASIL, 2008). Esse princípio vem sendo aplicado e promovido desde sua idealização em 1959 e, ainda assim, não é conhecido por todos aqueles que fazem experimentos. Os dados indicam que ainda é necessária a divulgação e educação das equipes sobre a premissa dos 3Rs.

O enriquecimento ambiental (EA) é um tópico conhecido por grande parte dos participantes (48; $77,4 \%$ ), que conceituaram o termo corretamente $(33 ; 80,5 \%)$. O EA é um princípio de manejo que aumenta a qualidade de vida dos animais cativos, identificando e fornecendo estímulos para a expressão de atividades físicas e psicológicas necessárias para seu bem-estar (Shepherdson, 1998). Sua utilização é indicada como favorável, mas não obrigatória, no manejo dos animais usados em laboratórios (Canadian Council on Animal Care, 1999; Baumans, 2010), de forma que sua aplicação ainda não integra a rotina de muitos laboratórios e biotérios. No Brasil, a Resolução Normativa $\mathrm{n}^{\circ} 12$, de 20 de setembro de 2013, do Concea - Conselho Nacional de Controle de Experimentação Animal (BRASIL, 2013) passa a exigir que o EA seja considerado na manutenção de animais em laboratório. Adicionalmente, os resultados mostram interesse na participação de um projeto de EA por parte dos entrevistados $(34 ; 69,4 \%)$. O EA, além de melhorar o grau de bem-estar, promove o comportamento natural das espécies e diminui a ocorrência de comportamentos anormais (Kastelein e Wiepkema, 1989), os quais podem interferir nos resultados (Bohannon, 2002). A abertura dos respondentes para o estudo de EA pode facilitar a exposição e a discussão com a 
comunidade científica acerca da ampliação de sua adoção.

Os participantes indicaram saber o significado de BEA (56; 90,3\%). Todavia, a maioria das conceituações foi parcial $(32 ; 64,0 \%) ; 19,4 \%$ (12) foram insatisfatórias; e somente $9,7 \%$ (seis) foram satisfatórias. Em estudos realizados com a população da Austrália, o termo "bom BEA" foi indicado por metade dos participantes $(n=500)$ como sendo a prevenção da crueldade e o tratamento humanitário dos animais, uma definição muito semelhante ao conceito apresentado pelos participantes desta pesquisa. Ainda que haja diferentes definições na literatura, há relativo consenso de que o conceito de BEA deve envolver questões físicas, comportamentais e psicológicas, conforme proposto em Webster (2005). Com relação ao conhecimento do conceito das cinco liberdades que constituem o BEA, 46,8\% (29) afirmaram conhecê-lo, dos quais $30,6 \%$ (19) conceituaram satisfatoriamente, enquanto $3,2 \%$ (dois) conceituaram parcialmente e $8,1 \%$ (cinco) responderam de forma insatisfatória. Os resultados sugerem uma necessidade de ampliar a educação em BEA.

Disciplinas relacionadas ao BEA estão disponíveis em um terço das instituições que mantêm curso de Medicina Veterinária no Brasil, sendo a oferta, em sua maioria, por meio de disciplinas optativas (Cortes de Lima et al., 2014). Apesar de o ensino de BEA no Brasil estar em crescimento nas escolas de Medicina Veterinária e Zootecnia (Cortes de Lima et al., 2014), é provável que ele não ocorra amplamente em cursos de Biologia, Farmácia, Medicina, Psicologia e outros que utilizam animais, como sugere a ausência de publicações abordando esse tema. Isso talvez ocorra devido ao fato de a ciência do BEA estar atrelada historicamente às ciências agrárias. Tendo em vista que apenas $38,7 \%$ (28) dos respondentes possuem formação em ciências agrárias, parece haver uma importante justificativa para que o ensino de BEA seja inserido em outros cursos de graduação envolvidos com o uso de animais.
Em relação à senciência, $70,0 \%$ (42) dos respondentes indicaram conhecer o termo. O significado da senciência animal foi corretamente exposto por $51,6 \%$ (32) dos participantes e de maneira imparcial por $12,9 \%$ (oito). Os valores atribuídos pelos respondentes ao grau de senciência foram maiores para mamíferos, sequencialmente decrescendo nas aves, peixes e invertebrados (Fig.1). Seguindo concentração decrescente de frequência da nota máxima, o grau de senciência indicado pelos respondentes em relação ao cão, ao bebê humano, ao lobo, ao porco, ao camundongo e ao rato não apresentou diferença (Fig.1); o grau de senciência atribuído ao frango não diferiu de todos os citados anteriormente, exceto do cão. $\mathrm{O}$ grau de senciência atribuído ao peixe não apresentou diferença em relação ao frango, ao caranguejo e à borboleta, os quais não diferiram entre si. É possível verificar um ponto de transição da percepção da senciência com base nas respostas relativas aos peixes; a partir delas, tem-se mais da metade das opiniões dispersas entre as notas 1 e 9. Os resultados evidenciam uma dificuldade dos respondentes em perceber a senciência dos peixes.

Os vertebrados são considerados sencientes devido às semelhanças biológicas com os organismos que podem vivenciar sentimentos (Braithwaite e Boulcott, 2007). Uma das possibilidades do baixo reconhecimento da senciência para os peixes são as questões culturais, que não são estritamente influenciadas pela ciência e por vezes tardam a ser atualizadas. As baixas notas e sua maior variação para invertebrados têm relação com o pensamento científico, em razão das diferenças anatômicas e da falta de conhecimento sobre potenciais estruturas nervosas capazes de gerenciar sentimentos em animais invertebrados, caracterizando incerteza epistêmica. Entretanto, há consenso entre neurologistas eminentes de que alguns invertebrados são sencientes (Low et al., 2012). 
Deguchi et al.
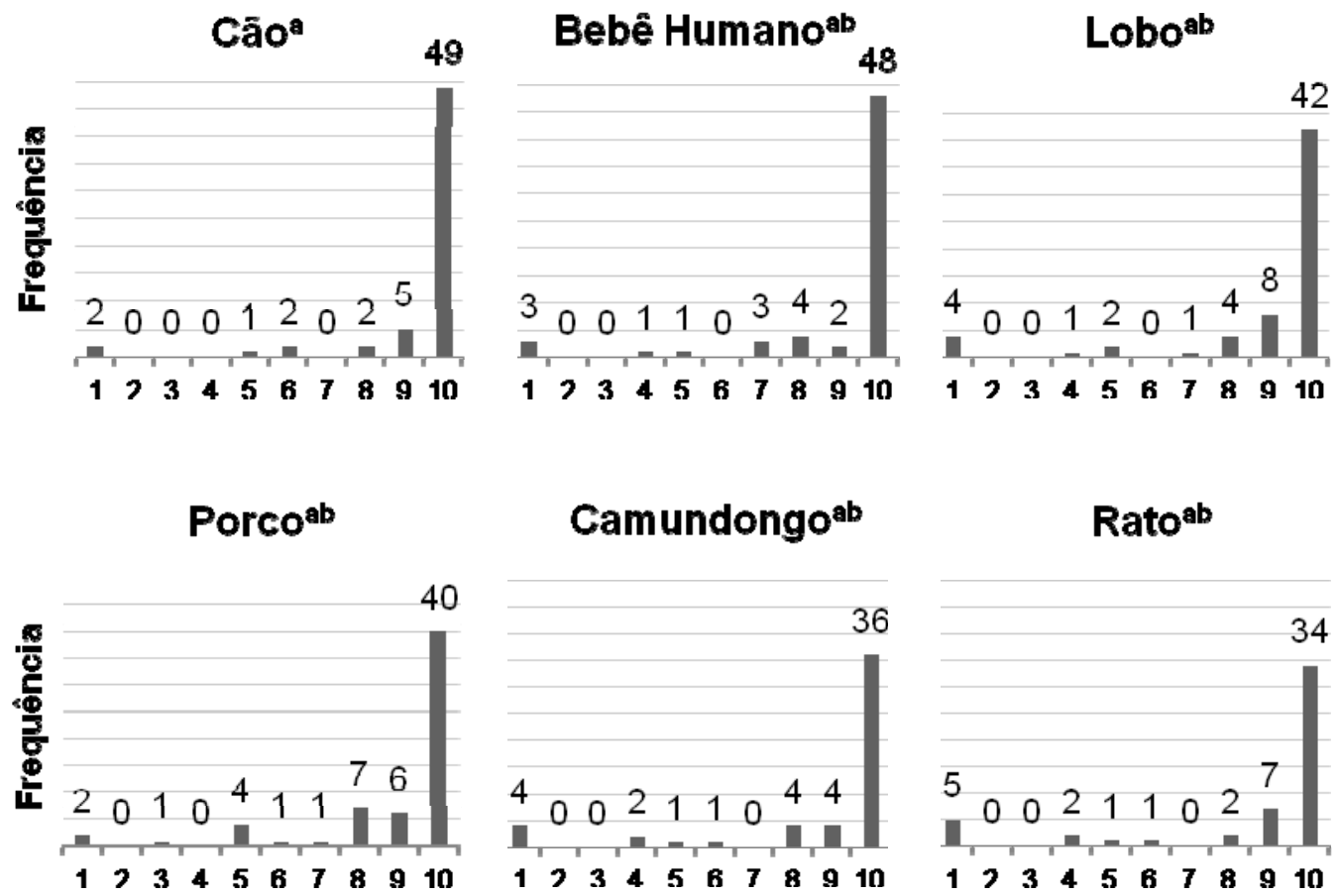

Frangobc

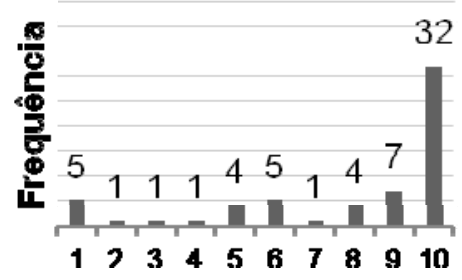

32

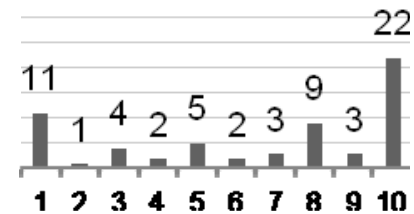

22

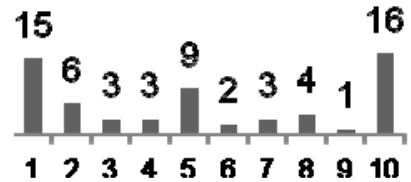

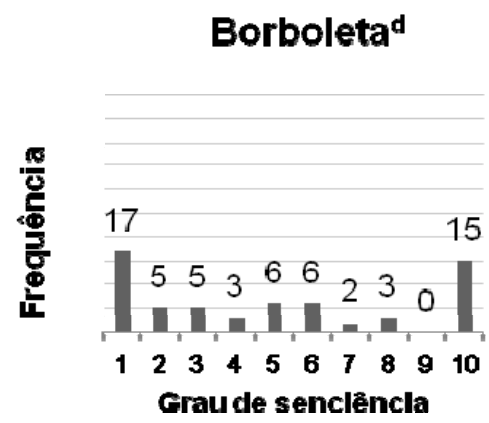

Figura 1. Frequência das notas e comparação quanto à percepção de grau de senciência seguindo-se a concentração decrescente de frequências da nota máxima. Grupos com letras iguais não apresentam diferenças significativas no teste de Mann-Whitney a $0,05 \%$, e grupos com letras diferentes apresentam diferenças significativas. 
Os resultados indicam que as equipes laboratoriais reconhecem $\mathrm{o}$ alto grau de senciência de vários grupos de animais, de tal forma que os consideram tão capazes de sentir quanto seres humanos imaturos. Segundo Driscoll (1995), alguns animais são julgados como portadores de maiores capacidades de inteligência e responsividade que os seres humanos. A proximidade social do ser humano com o cão pode fazer com que sua percepção seja influenciada por fatores emocionais. O mesmo pode ser exposto quanto aos ratos e camundongos que estão em constante contato com as equipes laboratoriais, acompanhados diariamente em suas rotinas. Além disso, o próprio fato de se estar trabalhando com seres vivos que são utilizados para compreensão de fenômenos nos seres humanos pode trazer, intrinsecamente, a proposta da semelhança biológica.

\section{CONCLUSÃO}

O BEA é uma ciência que ainda necessita de expansão nos ambientes que envolvem a utilização de animais em laboratório, pois poucos laboratoristas têm conhecimento satisfatório nessa área. São persistentes as dúvidas sobre as funções das CEUAs e a falta de treinamento em relação às ciências de animais em laboratório. No entanto, há preocupação dos laboratoristas com o sofrimento animal, associada à percepção de mais alto grau de senciência nas espécies que apresentam maior vínculo com os seres humanos, incluindo interesse em EA. Os resultados apresentados podem subsidiar a localização de pontos críticos relacionados à percepção em relação a questões de BEA em laboratórios brasileiros, apontando como caminhos imediatos à implantação de EA e o investimento em educação em bem-estar de animais usados em laboratórios.

\section{AGRADECIMENTOS}

Os autores agradecem à Capes - Coordenação de Aperfeiçoamento de Pessoal de Nível Superior, pela concessão de bolsa de estudos; à Ana Maria Aparecida Guaraldo, da Unicamp, SP; a equipe do Labea - Laboratório de Bem-estar Animal/UFPR; e a todos os respondentes.

\section{REFERÊNCIAS}

BAUMANS, V. Science-based assessment of animal welfare: laboratory animals. Rev. Sci. Tech., v.24, p.503-513, 2005.

BAUMANS, V. The laboratory mouse. In: The UFAW handbook on the care and management of laboratory and other research animals. 8.ed. Oxford, UK: Blackwell Publishing, 2010. p.276310 .

BEAGLE FREEDOM PROJECT. 2012 Disponível em: $<$ http://www.beaglefreedomproject.org $>$ Acessad o em: 18 abr. 2012.

BOHANNON, J. Can a mouse be standardised? Science, v.298, p.2320-2321, 2002.

BRAITHWAITE, V.A.; BOULCOTT, P. Pain perception, aversion and fear in fish. Dis. Aquat. Organ., v.75, p.131-138, 2007.

BRASIL. Presidência da República. Lei $n^{\circ}$ 11.794, 8 de outubro de 2008. Regulamenta o inciso VII do $\S 1$. do art. 225 da Constituição Federal, estabelecendo procedimentos para o uso científico de animais; revoga a Lei n.6.638, de 8 de maio de 1979; e dá outras providências. Diário Oficial da União, Brasília, 9 de outubro de 2008. Seção 1, p.1-4.

BRASIL. Conselho Nacional de Controle de Experimentação Animal. Resolução Normativa n.12, de 20 de setembro de 2013. Dispõe sobre o cuidado e a utilização de animais para fins científicos e didáticos. Diário Oficial da União. Brasília, 25 de setembro 2013. Disponível em: $<$ http://www.mct.gov.br/upd_blob/0228/228352. pdf>. Acessado em: 14 abr. 2015.

CORTES DE LIMA, M.; STAMM, F.O.; CAPILE, K.V.; GEBARA, R.R. et al. Ensino de Bem-estar Animal no Brasil. In: PRECIADO, J.J.T. (Org.). Bienestar animal y educación veterinaria en latinoamérica: diagnóstico. Guadalajara: Universidad de Guadalajara, 2014. v.1, p.27-39.

CRESSEY, D. Battle scars. Nature, v.470, p.452-453, 2011.

DEGUCHI, B.G.F.; MOLENTO, C.F.M.; DE SOUZA, C.E.P. The perception of students on the use of animals in higher education at the Federal University of Paraná, Southern Brazil. Altern. Lab. Anim., v.40, p.83, 2012. 
DRISCOLL, J.W. Attitudes toward animals: species ratings. Soc. Anim., v.3, p.139-150, 1995.

FAITH, R,E.; HUERKAMP, M.J. Environmental considerations for research animals. In: HESSLER, J.R.; LEHNER, N.D.M (Eds.) Planning and designing research animal facilities. San Diego, CA: Academic Press, 2009. p.59-83.

GUIDELINES on institutional animal user training. Canadian Council on Animal Care 1999. Disponível em: <http://www.ccac.ca/ Documents/Standards/Guidelines/Institutional_tr aining.pdf $>$ Acessado em: 22 abr. 2013.

KASTELEIN, R.A.; WIEPKEMA, P.R. A digging through as occupational therapy for Pacific Walrusses (Odobenus rosmarus divergens) in human care. Aquat. Mamm., v.15, p.9-17, 1989.

LOW, P.; PANKSEPP; J.; REISS, D. et al. The Cambridge declaration on consciousness, 2012. Disponível em:

http://fcmconference.org/img/CambridgeDeclara tionOnConsciousness.pdf $>$ Acessado em: 16 jul. 2013.

NEVALAINEN, T. Research, animals and welfare. Regulations, alternatives and guidelines. In: KALISTE, E. (Ed.) The welfare of laboratory animals. Dordrecht, The Netherlands: Springer, 2007. p.15-22.
SCHUPPLI, C.A.; FRASER, D. Factors influencing the effectiveness of research ethics committees. J. Med. Ethics, v.33, p.294-301, 2007.

SHEPHERDSON, D.J Tracing the path of environmental enrichment in zoos. In: SHEPHERDSON, D.J.; MELLEN, J.D.; HUTCHINS, M. (Eds.). Second nature: environmental enrichment for captive animals. California: Smithsonain Books, 1998. p.1-12.

SILLA, V.C.B.; SANS, E.C.O.; MOLENTO, C.F.M. An estimation of the extent of animal use in research in Brazil, as determined by bibliographic sampling from journals published in the State of Paraná. Altern. Lab. Anim., v.38, p.29, 2010.

TAYLOR, K.; GORDON, N.; LANGLEY, G.; HIGGINS, W. Estimates for worldwide laboratory animal use in 2005. Altern. Lab. Anim., v.36, p.327-342, 2008.

TREZ, T.A. Experimentando a desumanização: Paulo Freire e o uso didático de animais. Rev. Bras. Educ. Cient. Tecn., v.4, p.50-66, 2011.

ZANETTI, M.B.F.; SCHMIDT, E.M.S.; GARCIA, T.B. Recursos didáticos utilizados nas aulas práticas do curso de Medicina Veterinária em universidades do Estado do Paraná. Rev. Cient. Med. Vet., v.9, p. 351-360, 2011.

WEBSTER, J. Animal welfare: limping towards. 2.ed. Oxford, UK: Blackwell, 2005. 